\title{
Pathophysiologie der Psoriasis
}

\section{Pathophysiology of Psoriasis}

\section{Zusammenfassung}

Erkenntnisse zur (Immun-)Pathogenese der Psoriasis haben einerseits kürzlich zur Einführung mehrerer Pathogenese-orientierter Therapeutika geführt; andererseits haben diese neuen Therapien auch der Psoriasisforschung einen beispiellosen Aufschwung beschert. Insbesondere die Rolle entzündlicher Mediatoren wie Zytokine und Chemokine, aber auch die Funktion von Adhäsionsmolekülen bei der Rekrutierung von Immunzellen wurden intensiv beforscht. Diese Facetten müssen jedoch, und das wird gerade durch neueste Arbeiten verdeutlicht, durch weitere Bausteine eines komplexen Mosaikes, welches auch epidermale und neuronale Faktoren beinhaltet, ergänzt werden. Die komplexen Zusammenhänge der Pathophysiologie der Psoriasis werden in dieser Übersicht diskutiert.
Abstract

On the one hand, new insights into the (immuno-)pathogenesis of psoriasis have recently resulted in the approval of several pathogenesis-oriented novel therapeutics. These new therapies, on the other hand, have greatly stimulated research into the pathogenesis of psoriasis. In particular, pro-inflammatory mediators such as cytokines and chemokines as well as adhesion molecules involved in recruitment of immune cells into the skin have been in the focus of psoriasis research. However, new results indicate that the pathogenic mosaic of psoriasis has to be complemented by other important factors, such as epithelial or neurological dysregulations. This review discusses aspects of the complex pathophysiology of psoriasis.
Kaum eine andere dermatologische Erkrankung ist in der vergangenen Dekade derart in das Zentrum sowohl des klinischen als auch des wissenschaftlichen Interesses gerückt wie die Psoriasis [1]. Dies beruht einerseits darauf, dass die Erkenntnis, dass fehlgesteuerte Immunreaktionen eine wesentliche Rolle bei der Entstehung der Erkrankung spielen, in neue und selektive Therapieverfahren umgesetzt werden konnte. Andererseits hat gerade die Wirksamkeit verschiedener immunmodulatorisch wirksamer Therapien die Rolle des Immunsystems bei der Pathogenese der Psoriasis untermauert $[2,3]$. Wegen der leichten
Zugänglichkeit der typischen Läsionen an der Haut ist die Psoriasis heute geradezu eine „Modellerkrankung“ zum Studium immunpathogenetischer Vorgänge, aber auch für die klinische Entwicklung pathogeneseorientierter Therapiestrategien geworden. Allerdings deuten neueste Untersuchungen auch wieder stärker darauf hin, dass die primäre pathogenetische Rolle des Immunsystems durch andere Faktoren, beispielsweise epitheliale oder neuronale Fehlregulationen, ergänzt werden muss [4]. 
Das „klassische“ Konzept der Immunpathogenese der Psoriasis

Zahlreiche klinische und experimentelle Daten deuten darauf hin, dass dysregulierte T-Lymphozyten eine ganz wesentliche pathogenetische Rolle bei der Psoriasis spielen [5]. Diese Daten umfassen beispielsweise das therapeutische Ansprechen der Psoriasis auf Cyclosporin A, ein vorwiegend (allerdings nicht ausschließlich) auf T-Zellen wirkendes Immunsuppressivum [6]. Tatsächlich war die Beobachtung, dass Cyclosporin A die Psoriasis bessern kann, ein wesentlicher Baustein zur Etablierung des immun-pathogenetischen Konzeptes, welches später durch weitere Therapien, die primär durch Hemmung von T-Lymphozyten wirken, untermauert wurde. $\mathrm{Zu}$ letzteren gehören beispielsweise das Toxin DAB ${ }_{389} \mathrm{IL}-2$ [7] oder Antikörper gegen CD2 [8], CD11a $[9,10]$ oder CD4 $[11,12]$. Einige dieser Prinzipien sind bereits in zugelassene Therapien umgesetzt worden (beispielsweise Efalizumab (Raptiva ${ }^{\circledR}$ ) und Alefacept (Amevive ${ }^{\circledR}$ ), letzteres allerdings in Deutschland nicht zugelassen). Ein so genannter „PsoriasisSuzeptibilitäts-Lokus“ im Genom ist mit einem Gen assoziiert, welches IL-2, einen wesentlichen T-Zell-Wachstumsfaktor, reguliert [13]. Kasuistisch wurde berichtet, dass die Psoriasis nach Knochenmark-Transplantationen beim Empfänger neu auftrat bzw. abheilte, je nach Konstellation von Spender und Empfänger $[14,15]$. Auch die Assoziation der Psoriasis mit bestimmten MHC-Allelen, beispielsweise -B13, -B17, -Bw57 und -Cw6 legt eine pathogene Rolle von T-Zellen nahe [16]. Manche Arbeitsgruppen haben sogar berichtet, dass in psoriatischen Läsionen oligoklonale T-Zell-Proliferation auftritt, was eine antigenspezifische T-Zell-Antwort nahe legt [17-19]. Der in manchen Fällen fehlende Nachweis oligoklonaler T-Zell-Expansion könnte an der Kolonisation psoriatischer Läsionen mit Superantigen-produzierenden Bakterien liegen [20,21]. Die Rolle bakterieller Superantigene als Auslöser (Provokationsfaktoren) psoriatischer Läsionen ist inzwischen recht gut etabliert $[20,22,23]$. Darüber hinaus wurde spekuliert, dass auf Grund von Sequenzhomologien zwischen M-Peptiden von Stretokokken und humanen Intermediärfilamenten (z. B. Keratin 17) Strukturproteine als (Auto-)Antigene bei der Psoriasis fungieren könnten [24].

Obwohl die Forschung zur Pathogenese der Psoriasis wesentlich durch den Mangel an adäquaten Tiermodellen erschwert wird, sprechen zahlreiche tierexperimentelle Befunde ebenfalls für eine zentrale Beteiligung der T-Zellen an der Pathogenese der Psoriasis [25]. In Xenotransplantations-Modellen führte die Injektion von T-Lymphozyten psoriatischer Spender zur Ausbildung psoriatischer Gewebeveränderungen in den Transplantaten [26]. Bakterielle Superantigene können auch in diesen Modellen die Funktion der pathogenen T-Zellen verstärken [23]. Ein neueres Transplantationsmodell mit RAG-2 (recombination activating protein-2-)defizienten Mäusen impliziert darüber hinaus, dass in bestimmten Fällen residente T-Zellen für die Ausbildung psoriatischer Läsionen ausreichen können [27]. Schließlich wurden Tiermodelle beschrieben, in denen allein durch adoptiven Transfer bestimmter T-Zell-Populationen und ohne primäre kutane Veränderungen Psoriasis-ähnliche Phänotypen induziert werden konnten $[28,29]$.
Selbstverständlich sind auch verschiedene andere Zellen des Immunsystems, wie neutrophile Granulozyten, Makrophagen und Mastzellen, an der Pathogenese der Psoriasis beteiligt [30,31] und in manchen Tiermodellen kann durch gezielte Beeinflussung dieser Zellen eine Besserung Psoriasis-ähnlicher Hautveränderungen erreicht werden [32]. Verschiedene antipsoriatische Medikamente, wie beispielsweise Fumarsäureester, entfalten ihre Wirkung (zumindest teilweise) auch über eine Modulation neutrophiler Granulozyten und anderer Immunzellen [33]. Allerdings sind Mechanismen, welche eine primäre Rolle anderer Zellen des Immunsystems belegen, bislang nicht beschrieben worden und es bestehen hier sicherlich vielfältige Wechselwirkungen mit T-Lymphozyten [34,35].

\section{Zytokine und Chemokine als Mediatoren der Psoriasis}

Psoriasis ist ein „Paradebeispiel“, in welchem die komplexen Wechselwirkungen verschiedener löslicher und gebundener Zytokine und Chemokine bei der Pathogenese einer chronisch-entzündlichen Erkrankung deutlich werden [36,37]. Dabei können die bisher bekannten Funktionen beteiligter Mediatoren die psoriatischen Gewebeveränderungen zumindest teilweise erklären (Abb.1): Beispielsweise kann durch TNF $\alpha$, IL-1, IL-6 und TGF $\alpha$ die Expression verschiedener für die Einwanderung von Immunzellen relevanter Adhäsionsmoleküle stimuliert werden [38 - 40]. Insbesondere die Rolle von TNFo, welches als „primäres" Zytokin die Sekretion weiterer Mediatoren anregt, ist intensiv beforscht und bereits in zugelassene Therapien umgesetzt worden (derzeit zugelassen sind Etanercept $\left(\right.$ Enbrel $\left.^{\circledR}\right)$ und Infliximab (Remicade ${ }^{\circledR}$ ); Adalimumab (Humira ${ }^{\circledR}$ ) ist zur Therapie der Psoriasis-Arthritis zugelassen) [3,9,41,42]. Zur Ausprägung des psoriatischen Phänotyps tragen sicherlich auch das Angiopoetin-System [43] sowie weitere angiogene Faktoren, wie VEGF (vascular endothelial growth factor) [44] bei. Ähnliches gilt für die Dysregulation immunsuppressiver Zytokine, beispielsweise IL-10 [45], eine Hypothese, welche durch den therapeutischen Effekt von IL-10 bei manchen Psoriasis-Patienten gestützt wird [46]. Ein Th1-gewichtetes Zytokinprofil, in welchem IFN $\gamma$, IL-2 sowie durch diese induzierte weitere Zytokine wie IL-12 und IL-17 über Th2-Zytokine, beispielsweise IL-4 und -5 dominieren, ist für die Psoriasis typisch [47]. Entsprechend kann eine Verschiebung dieses Milieus therapeutisch ausgenutzt werden $[46,48,49]$. Die genauen Mechanismen der Zytokin-Feinregulation im psoriatischen Mikromilieu werden allerdings noch nicht genau verstanden.

Chemokine haben zentrale Aufgaben bei der gewebespezifischen Rekrutierung von Immunzellen und sind deshalb seit einigen Jahren in den Blickpunkt der Psoriasis-Forschung gelangt [37,50]. Zu den Chemokinen, welche an der Rekrutierung von Leukozyten in die Haut beteiligt sind, gehören TARC (thymus and activation regulated chemokine) und MDC (macrophage-derived chemokine), welche von den dermalen Blutgefäßen exprimiert werden und über den CC-Chemokin-Rezeptor CCR4 wahrscheinlich CLA-(cutaneous lymphocyte-associated antigen-)exprimierende T-Zellen in die Haut rekrutieren [51]. Darüber hinaus spielt das CC-Chemokin CCL27 (CTACK, cutaneous T cell-attracting chemokine), ein Ligand für CCR10, eine wichtige Rolle bei der epidermalen T-Zell-Rekrutierung [52]. Weitere Beispiele 


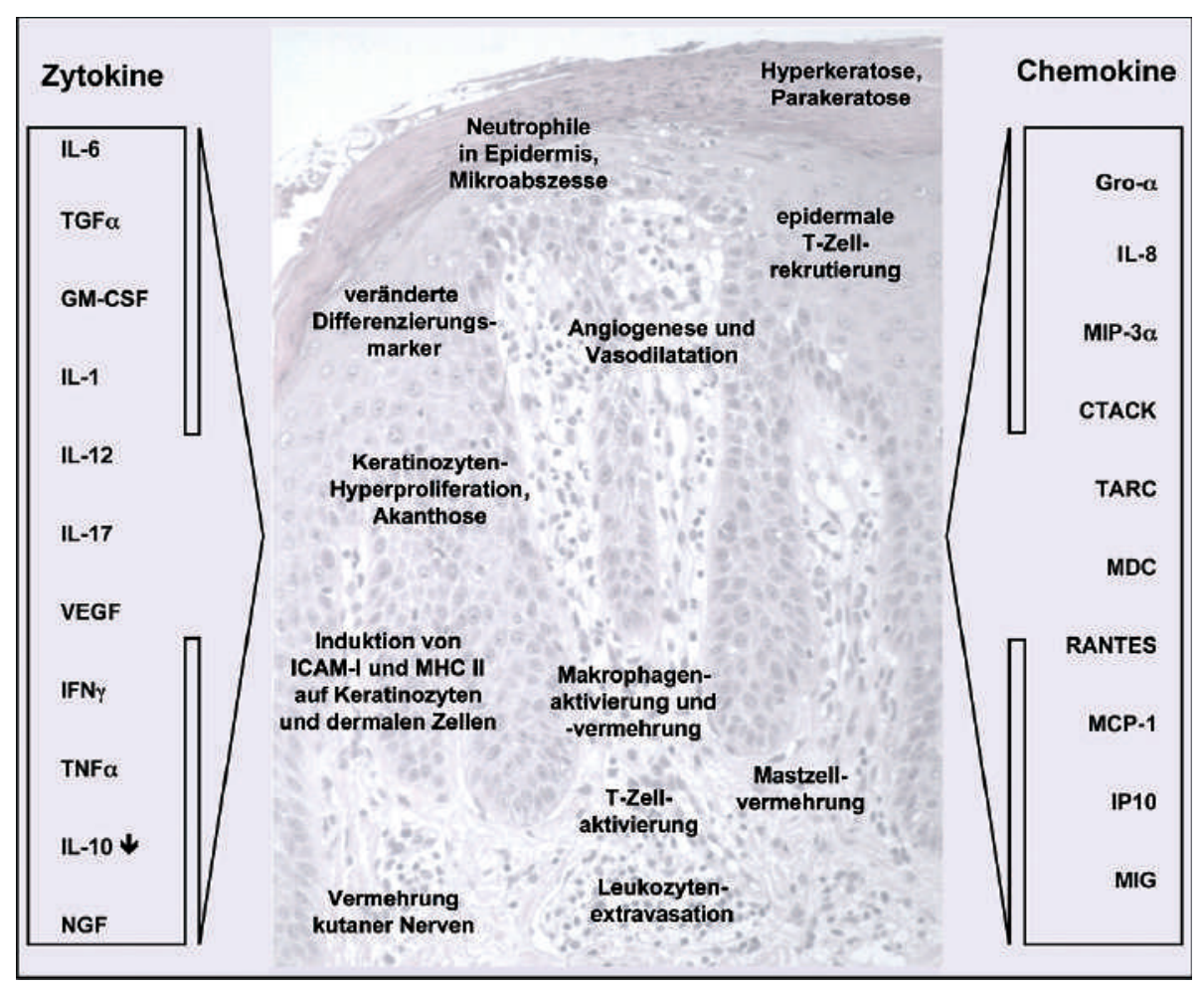

Abb. 1 Komplexe Wechselwirkungen zahlreicher Mediatoren in psoriatischer Haut (modifiziert nach [3]). Die Abbildung zeigt schematisch ausgewählte Mediatoren (Zytokine auf der linken und Chemokine auf der rechten Seite), für die gezeigt wurde, dass sie in psoriatischer Haut fehlreguliert werden und die einzeln oder in Kombination die erwähnten sowie weitere Gewebeveränderungen bewirken können.

für Chemokine, die wahrscheinlich in die Pathogenese der Psoriasis involviert sind, sind MIP-3 $\alpha$ (macrophage inflammatory protein-3 $\alpha$ ) und MIG (monokine induced by IFN $\gamma$ ) [53]. Da MIG, ein chemotaktischer Faktor für T Zellen, in Makrophagen und Endothelzellen durch IFN $\gamma$ induziert wird [54], liegt hier möglicherweise eine pro-inflammatorische, T-Zell-assoziierte positive Rückkopplung im psoriatischen Mikromilieu vor. Diese Aktivität kann durch weitere Chemokine, beispielsweise RANTES (regulated on activation, normal T-cell expressed and secreted) und MCP-1 (monocyte chemotactic protein-1), welche ebenfalls in psoriatischer Epidermis hoch exprimiert werden, verstärkt werden $[55,56]$. MCP-1 und RANTES können auch Mastzellen in die psoriatische Haut attrahieren [55].

Im Zusammenhang mit Psoriasis muss auch IL-8 (CXCL8) erwähnt werden, das als eines der ersten Chemokine in psoriatischer Haut beschrieben wurde [57]. IL-8 vermittelt durch seine Rezeptoren CXCR1 und CXCR2 die Rekrutierung neutrophiler Granulozyten. Allerdings führte die Antikörper-vermittelte IL8-Hemmung nicht zu signifikanter Besserung der Psoriasis in einer klinischen Studie [50], ein Indiz für die Redundanz und Komplexität des Chemokin-Systems. Insgesamt scheinen viele Funktionen von Chemokinen und ihrer Rezeptoren überlappend bzw. redundant reguliert zu sein. Dies ist wahrscheinlich ein wichtiger Grund dafür, dass bislang noch keine Chemokin-gerichteten Therapien bis zur Marktreife entwickelt werden konnten [58].

Die Komplexität der Psoriasis-Pathogenese wird auch dadurch unterstrichen, dass offenbar ganz unterschiedliche Zellen und deren Botenstoffe hierin involviert sind. $\mathrm{Zu}$ diesen auf den ersten Blick nicht erwarteten Mechanismen gehört beispielsweise die Beteiligung des (kutanen) Nervensystems. Bereits vor mehr als dreißig Jahren wurde berichtet, dass Blockade oder Verletzung peripherer Nerven zur Abheilung der Psoriasis im versorgten Ge- biet führen kann $[59,60]$. Später wurden die Induktion verschiedener Neuropeptide (beispielsweise substance P und CGRP (calcitonin gene-related protein)) sowie die Vermehrung kutaner Nerven in psoriatischen Läsionen beschrieben [61]. Diese sowie weitere Beobachtungen führten zu der Hypothese, dass auch neuro-immunologische Mechanismen in die Pathogenese der Psoriasis involviert sind, was durch kürzlich beschriebene Funktionen von NGF (nerve growth factor) und seines Rezeptors (p75 Neurotrophin-Rezeptor) sowie Tyrosin-Kinase A erhärtet wurde $[62,63]$. Zumindest in einem präklinischen Tiermodell konnten psoriatische Hautveränderungen bereits durch Antikörper-vermittelte Blockade des NGF-Rezeptors signifikant gebessert werden [64].

\section{Adhäsionsmolekuille bei Psoriasis}

Wie bei sämtlichen entzündlichen Erkrankungen, wandern auch in psoriatischer Haut Leukozyten in genau festgelegten Schritten in das entzündete Gewebe ein $[58,65]$. Diese Schritte werden von den bereits erwähnten Zytokinen und Chemokinen, vor allem aber auch von einer recht großen Zahl von Adhäsionsmolekülen reguliert und geleitet, durch welche Zellen des Immunsystems - gleichsam wie auf einer molekularen Leiter - in die Haut gelangen. Wirklich Psoriasis-spezifische Schritte scheinen hierbei nicht zu existieren; dennoch ist die Psoriasis vielfach als Modell für grundlegende Vorgänge bei der Leukozyten-Rekrutierung genutzt worden $[9,66]$. Eines der in diese RekrutierungsSequenz involvierten Adhäsionsmoleküle, das Integrin CD11a/CD18, wird bereits klinisch als therapeutische Zielstruktur zur Behandlung der Psoriasis durch den monoklonalen Antikörper Efalizumab genutzt [10,67]. 


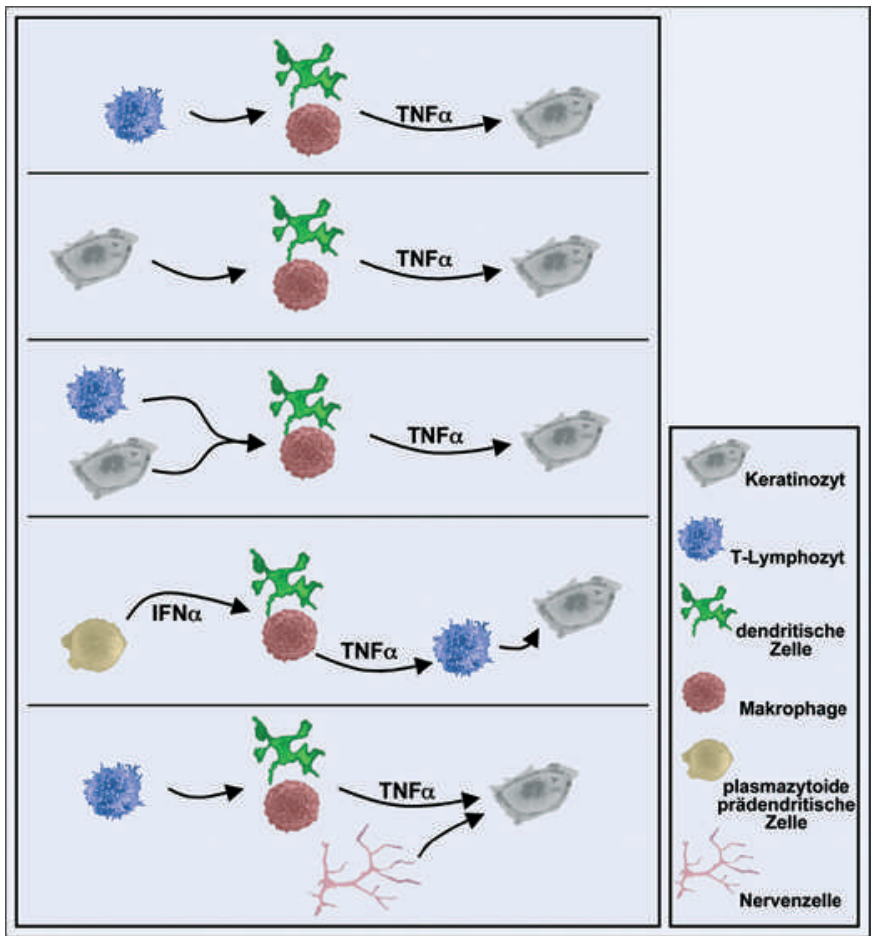

Abb. 2 Ausgewählte mögliche Szenarien der Initiierung der Pathogenese-Kaskade der Psoriasis (modifiziert und erweitert nach [4]). Favorisert wird heute von den meisten Forschern noch eine primär T-Zell-induzierte Immunpathogenese der Psoriasis (oben). Einige klinische Beobachtungen und neuere Tiermodelle sprechen jedoch dafür, dass auch primär keratinozytäre Veränderungen (2. von oben), eine Kombination aus T-Zell- und Keratinozyten-Induktion (3. von oben), Antigenpräsentierende Zellen (4. von oben) und/oder kutane Nervenzellen (unten) wichtige Funktionen bei der Initiierung der Pathogenese-Kaskade haben könnten.

Die ersten Schritte der Leukozyten-Rekrutierung aus der Zirkulation, das lockere Anheften und das Rollen an der Gefäßwand, werden durch Adhäsionsmoleküle aus der Selektin-Familie vermittelt. Diese Rezeptoren binden an Zuckerstrukturen (beispielsweise Sialyl-Lewis $\left.{ }^{\mathrm{X}}\right)$, welche auf Proteingerüsten der jeweils adhärierenden Zellen präsentiert werden $[65,68,69]$. Aktivierte Endothelzellen exprimieren E-Selektin (CD62E) und P-Selektin (CD62P), während Leukozyten L-Selektin (CD62L) exprimieren [70]. Lymphozyten exprimieren Selektin-Liganden, zu denen das speziell auf kutanen T-Zellen gefundene CLA (cutaneous lymphocyte-associated antigen) gehört [70,71].

Selektine zeigen ausgeprägte funktionelle Überlappungen. Wahrscheinlich ist dies ein Grund, aus dem die Blockade einzelner Selektine nicht zu ausreichender Besserung der Psoriasis in klinischen Studien geführt haben $[72,73]$. Substanzen, welche gleichzeitig mehrere Selektine blockieren, könnten hier vorteilhafter sein [74-78].

Die auf das Rollen folgende feste Adhäsion und Extravasation von Lymphozyten wird - nach Aktivierung durch Chemokin-Rezeptoren - insbesondere durch Adhäsionsmoleküle aus der IntegrinFamilie vermittelt. Integrine sind heterodimere TransmembranRezeptoren, welche meist an Komponenten der extrazellulären Matrix und/oder membranständige Adhäsionsmoleküle aus der Immunglobulin-Superfamilie (beispielsweise ICAM-1 (intercel- lular adhesion molecule-1), CD54) binden [79]. Vor allem $\beta 2$ und $\beta 1$-Integrine sind für die feste Adhäsion von Leukozyten am Gefäßendothel wichtig. Die gezielte Blockade des $\beta 2$-Integrins CD11a/CD18 wird (in Gestalt des monoklonalen Antikörpers Efalizumab [Raptiva $\left.{ }^{\circledR}\right]$ ) bereits erfolgreich zur Therapie der Psoriasis eingesetzt $[9,10,67]$. Verschiedene weitere Adhäsionsmoleküle, wie beispielsweise PECAM-1 (platelet endothelial cell adhesion molecule-1) oder Mitglieder der JAM-(junctional adhesion molecule-)Familie spielen ebenfalls wichtige Rollen bei der Lymphozyten-Extravasation [80,81].

Im Gegensatz zu unseren Kenntnissen zu Endothel-Adhäsion und Extravasation von Leukozyten wissen wir noch recht wenig über deren Rekrutierung in die Epidermis. In psoriatischer Epidermis ist beispielsweise die Expression von ICAM-1 induziert, welches als Ligand für einwandernde Leukozyten fungieren kann $[82,83]$. Auch $\beta 1$-Integrine scheinen für die epidermale Rekrutierung wichtig zu sein [84], obwohl dies noch nicht direkt gezeigt wurde. Durch seine Bindung an epidermales E-Cadherin [85] scheint auch das Integrin $\alpha \mathrm{E}(\mathrm{CD} 103) \beta 7$ die epidermale Rekrutierung mancher T-Zellen (besonders $\mathrm{CD}^{+}{ }^{+} \mathrm{T}$-Zellen) in psoriatische Epidermis zu vermitteln $[86,87]$.

\section{Primär kutane Fehlfunktionen als pathogenetisch relevante Faktoren?}

Obwohl in den vergangenen Jahren eine Vielzahl von Beobachtungen beschrieben wurden, welche eine primär immunologisch vermittelte Pathogenese der Psoriasis nahe legen, gibt es durchaus ernst zu nehmende Argumente, welche auf Fehlfunktionen residenter (dermaler und/oder epidermaler) Zellen als pathogenetischen Faktor hindeuten [4]. Die sich darauf gründende Debatte hat kürzlich insbesondere durch neue Tiermodelle Psoriasis-ähnlicher chronischer Hautentzündungen neue Nahrung erhalten:

So wurden vor wenigen Monaten genetisch veränderte Mäuse (transgene Mäuse) beschrieben, in welchen in der epidermalen Basalschicht die Transkriptionsfaktoren JunB sowie cJun induzierbar reduziert waren [88]. Diese Mäuse entwickelten eine chronische hyperproliferativ-entzündliche Hauterkrankung, die einige Ähnlichkeiten zur Psoriasis aufwies, sowie arthritische Gelenkveränderungen. Interessanterweise trat der Psoriasisähnliche Phänotyp an der Haut, weniger hingegen die Gelenkveränderungen, auch in JunB/cJun-Mutanten auf, welche RAG-2 (recombination activating protein-2-)defizient waren, also keine reifen T-Lymphozyten ausbilden können. Die Autoren der Arbeit folgerten (in Extrapolation auf die Psoriasis bei Menschen), dass T-Zellen nur eine sekundäre Rolle in der Pathogenese der Psoriasis spielen und dass vielmehr primär kutane Veränderungen im Vordergrund stehen. In einem anderen kürzlich publizierten Mausmodell wurde ein konstitutiv aktiver Transkriptionsfaktor, STAT3 (signal transducer and activator of transcription 3) in muriner Epidermis überexprimiert, was eine Verbindung eines primär von der Epidermis ausgehenden Psoriasis-ähnlichen Phänotyps mit T-Zell-Aktivierung nahe legt [89]. Damit reihen sie sich diese neueren Arbeiten ein in eine Anzahl von Argumenten, welche T-Lymphozyten zwar als beteiligte, aber nicht unbedingt als primär die Pathogenese-Kaskade auslösende Zellen sehen. Zu 
diesen Argumenten gehören strukturelle und biochemische Aberrationen in psoriatischen Keratinozyten [90], das therapeutische Ansprechen mancher psoriatischer Hautläsionen auf einen Parathormon-Rezeptor-Agonisten, der keine immunmodulatorische Aktivität hat, aber die Keratinozyten-Differenzierung normalisiert [91] oder die mögliche Initiierung einer inflammatorischen Kaskade durch Keratinozyten, die sich durch Komponenten der „angeborenen Immunität“ (innate immunity) mit Mikroorganismen auseinandersetzen und dadurch sekudär Immunzellen in die Haut rekrutieren [92]. Unter bestimmten experimentellen Bedingungen können auch plasmazytoide prä-dendritische Zellen in Abhängigkeit von TNF $\alpha$ und IFN $\alpha$ die „psoriatische Konversion“ von Hauttransplantaten im Tiermodell induzieren [93]. Schließlich sind auch die pathogenetische Rolle von Umweltfaktoren, beispielsweise mikrobieller (Super)-Antigene sowie die Provokation der Psoriasis durch bestimmte Medikamente oder Infektionen durch eine ausschließlich T-Zell-initiierte (d.h. Antigen-gerichtete) Pathogenese nicht hinreichend erklärbar.

Insgesamt scheint mir weiterhin, dass die Evidenz für eine primär T-Zell-induzierte Pathogenese der Psoriasis sehr stark ist, obwohl wesentliche Faktoren, beispielsweise die Identifizierung eines (Auto-)Antigens noch ihrer Entdeckung harren. Vielleicht gelingt aber auch die Aufklärung genetischer Faktoren, die zumindest für einen Teil des psoriatischen Krankheitsspektrums charakteristisch sind. Allerdings bringen die Komplexität der Psoriasis-Pathogenese sowie die zahlreichen noch nicht gelösten Detailfragen es mit sich, dass die entscheidende Frage, ob nämlich eine primär immunologisch vermittelte oder doch eine auf primär kutanen Veränderungen beruhende Erkrankung vorliegt, noch nicht zweifelsfrei beantwortet werden kann. Grundsätzlich kann man immer noch mehrere pathogenetische Szenarien skizzieren, die sämtlich durch (mehr oder weniger) experimentelle Evidenz gestützt werden (schematisch zusammengefasst in Abb. 2). Die Forschung zur Pathogenese der Psoriasis scheint gleichsam wie ein Pendel [4] - zwischen Strömungen in Richtung primäre Immunpathogenese versus präexistenter kutaner Veränderungen zu schwingen. Zusammenfassend kann man deshalb wohl feststellen, dass die Forschung zur Pathogenese der Psoriasis weiterhin interessant und spannend bleiben wird; ihre letztliche Aufklärung kann dann vielleicht auch neue und selektive Therapien ermöglichen.

\section{Literatur}

${ }^{1}$ Schön MP, Boehncke WH. Psoriasis. N Engl J Med 2005; 352: 18991912

${ }^{2}$ Gottlieb AB. Psoriasis: emerging therapeutic strategies. Nat Rev Drug Discov 2005; 4: 19-34

${ }^{3}$ Schön MP. Advances in psoriasis therapy. Lancet 2005; 366: 1333 1335

${ }^{4}$ Nickoloff BJ. Keratinocytes regain momentum as instigators of cutaneous inflammation. Trend Mol Med 2006; 12: $102-106$

${ }^{5}$ Christophers E. The immunopathology of psoriasis. Int Arch Allergy Immunol 1996; 110: 199-206

${ }^{6}$ Mueller W, Herrmann B. Cyclosporin A for psoriasis. N Engl J Med 1979; 301: 555

${ }^{7}$ Gottlieb JL, Gilleaudeau P, Johnson R, Estes L, Woodworth TG, Gottlieb $A B$, Krueger JG. Response of psoriasis to a lymphocyte-selective toxin (DAB389 IL-2) suggests a primary immune, but not keratinocyte, pathogenic basis. Nature Med 1995; 1: $442-447$
${ }^{8}$ Ellis CN, Krueger GG, Group ACS. Treatment of chronic plaque psoriasis by selective targeting of memory effector T lymphocytes. N Engl J Med 2001; 345: 248-255

${ }^{9}$ Kupper TS. Immunologic targets in psoriasis. New Engl J Med 2003; 349: $1987-1990$

${ }^{10}$ Lebwohl M, Tyring SK, Hamilton TK, Toth D, Glazer S, Tawfik NH, Walicke P, Dummer W, Wang X, Garovoy MR, Pariser D. A novel targeted T-cell modulator, efalizumab, for plaque psoriasis. New Engl J Med 2003; 349: 2004-2013

${ }^{11}$ Nicolas JF, Chamchick N, Thivolet J, Wijdenes J, Morel P, Revillard JP. CD4 antibody treatment of severe psoriasis. Lancet 1991; 338: 321

12 Prinz J, Braun-Falco O, Meurer M, Daddona P, Reiter C, Rieber P, Riethmuller G. Chimaeric CD4 monoclonal antibody in treatment of generalized pustular psoriasis. Lancet 1991; 338: 320-321

${ }^{13}$ Tomfohrde J, Silverman A, Barnes R, Fernandez-Vina MA, Young M, Lory D, Morris L, Wuepper KD, Stastny P, Menter A, Bowcock A. Gene for familial psoriasis susceptibility mapped to the distal end of human chromosome 17q. Science 1994; 264: 1141 - 1145

${ }^{14}$ Eedy DJ, Burrows D, Bridges JM, Jones FGC. Clearance of severe psoriasis after allogeneic bone marrow transplantation. Br Med J 1990; 300 : 908

${ }^{15}$ Gardembas-Pain M, Ifrah N, Foussard C, Boasson M, Saint Andre JP, Verret JL. Psoriasis after allogeneic bone marrow transplantation. Arch Dermatol 1990; 126: 1523

${ }^{16}$ Christophers E. Psoriasis - Epidemiology and clinical spectrum. Clin Exp Dermatol 2001; 26: $314-320$

${ }^{17}$ Bour H, Puisieux I, Kouritsky P, Favrot M, Musette P, Nicolas JF. T-cell repertoire analysis in chronic plaque psoriasis suggests an antigenspecific immune response. Hum Immunol 1999; 60: 665-676

${ }^{18}$ Lin WJ, Norris DA, Achziger M, Kotzin BL, Tomkinson B. Oligoclonal expansion of intraepidermal T cells in psoriasis skin lesions. J Invest Dermatol 2001; 117: 1546-1553

${ }^{19}$ Prinz JC, Vollmer S, Boehncke WH, Menssen A, Laisney I, Trommler P. Selection of conserved TCR VDJ rearrangements in chronic psoriatic plaques indicates a common antigen in psoriasis vulgaris. Eur J Immunol 1999; 29: 3360-3368

${ }^{20}$ Boehncke W-H. Psoriasis and bacterial superantigens - formal or causal relation? Trends Microbiol. 1996; 4: 485-489

${ }^{21}$ Vekony MA, Holder JE, Lee AJ, Horrocks C, Eperon IC, Camp RD. Selective amplifications of T-cell receptor variable region species is demonstrable but not essential in early lesions of psoriasis vulgaris: analysis by anchored polymerase chain reaction and hypervariable region size spectratyping. J Invest Dermatol 1997; 109: 5-13

22 Weisenseel P, Laumbacher B, Besgen P, Ludolph-Hauser D, Herzinger T, Röcken M, Wank R, Prinz JC. Streptococcal infection distinguishes different types of psoriasis. J Med Genet 2002; 39: 767-768

${ }^{23}$ Boehncke W-H, Dressel D, Zollner TM, Kaufmann R. Pulling the trigger on psoriasis. Nature 1996; 379: 777

${ }^{24}$ Gudmundsdottir AS, Sigmundsdottir H, Sigurgeirsson B, Good MF, Valdimarsson $\mathrm{H}$, Jonsdottir I. Is an epitope on keratin 17 a major target for autoreactive T lymphocytes in psoriasis? Clin Exp Immunol 1999; 117: $580-586$

${ }^{25}$ Schön MP. Animal models of psoriasis - what can we learn from them? J Invest Dermatol 1999; 112: $405-410$

${ }^{26}$ Wrone-Smith T, Nickoloff BJ. Dermal injection of immunocytes induces psoriasis. J Clin Invest 1996; 98: $1878-1887$

${ }^{27}$ Boyman O, Hefti HP, Conrad C, Nickoloff BJ, Suter M, Nestle FO. Spontaneous development of psoriasis in a new animal model shows an essential role for resident $\mathrm{T}$ cells and tumor necrosis factor alpha. J Exp Med 2004; 199: 731 - 736

${ }^{28}$ Breban M, Fernandez-Sueiro JL, Richardson JA, Hadavand RR, Maika SD, Hammer RE, Taurog JD. T cells, but not thymic exposure to HLA-B27, are required for the inflammatory disease of HLA-B27 transgenic rats. J Immunol 1996; 156: 794-803

${ }^{29}$ Schön MP, Detmar M, Parker CM. Murine psoriasis-like disorder induced by naive CD4 ${ }^{+}$T-cells. Nature Med 1997; 3: 183-188

${ }^{30}$ Bos JD, de Rie MA, Teunissen MB, Piskin G. Psoriasis: dysregulation of innate immunity. Br J Dermatol 2005; 152: 1098 - 1107

${ }^{31}$ von den Driesch P. Is psoriasis a T-cell disease? Viewpoint 3. Exp Dermatol 2000; 9: 367-377

${ }^{32}$ Schön M, Denzer D, Kubitza RC, Ruzicka T, Schön MP. Critical role of neutrophils for the generation of psoriasiform skin lesions in flaky skin mice. J Invest Dermatol 2000; 114: 976-983 
${ }^{33}$ Zhu K, Mrowietz U. Enhancement of antibacterial superoxide-anion generation in human monocytes by fumaric acid esters. Arch Dermatol Res 2005; 297: 170-176

${ }^{34}$ Schaerli P, Britschgi M, Keller M, Steiner UC, Steinmann LS, Moser B, Pichler WJ. Characterization of human T cells that regulate neutrophilic skin inflammation. J Immunol 2004; 173: 2151 - 2158

${ }^{35}$ Keller M, Spanou Z, Schaerli P, Britschgi M, Yawalkar N, Seitz M, Villiger PM, Pichler WJ. T cell-regulated neutrophilic inflammation in autoinflammatory diseases. J Immunol 2005; 175: 7678 - 7686

${ }^{36}$ Nickoloff BJ. The cytokine network of psoriasis. Arch Dermatol 1991; 127: $871-884$

37 Schön MP, Ruzicka T. Psoriasis: The plot thickens. Nat Immunol 2001; 2: 91

38 Barker JNWN, Sarma V, Mitra RS, Dixit VM, Nickoloff BJ. Marked synergism between tumor necrosis factor-alpha and interferon-gamma in regulation of keratinocyte-derived adhesion molecules and chemotactic factors. J Clin Invest 1990; 85: 605-608

${ }^{39}$ Murphy JE, Robert C, Kupper TS. Interleukin-1 and cutaneous inflammation: a crucial link between innate and acquired immunity. J Invest Dermatol 2000; 114: 602-608

${ }^{40}$ Neuner P, Urbanski A, Trautinger F, Moller A, Kirnbauer R, Kapp A, Schopf E, Schwarz T, Luger TA. Increased IL-6 production by monocytes and keratinocytes in patients with psoriasis. J Invest Dermatol 1991; 97: 27-33

${ }^{41}$ Leonardi CL, Powers JL, Matheson RT, Goffe BS, Zitnik R, Wang A, Gottlieb $A B$, T.e.p.s.group. Etanercept as monotherapy in patients with psoriasis. N Engl J Med 2003; 349: 2014-2022

42 Reich K, Nestle FO, Papp K, Ortonne JP, Evans R, Guzzo C, Li S, Dooley LT, Griffiths CEM. Infliximab induction and maintenance therapy for moderate-to-severe psoriasis. Lancet 2005; 366: 1367 - 1374

${ }^{43}$ Kuroda K, Sapadin A, Shoji T, Fleischmajer R, Lebwohl M. Altered expression of angiopoietins and Tie2 endothelium receptor in psoriasis. J Invest Dermatol 2001; 116: 713-720

${ }^{44}$ Detmar M, Brown LF, Claffey KP, Yeo KT, Kocher O, Jackman RW, Berse B, Dvorak HF. Overexpression of vascular permeability factor/vascular endothelial growth factor and its receptors in psoriasis. J Exp Med 1994; 180: 1141 - 1146

${ }^{45}$ Michel G, Mirmohammadsadegh A, Olasz E, Jarzebska-Deussen B, Müschen A, Kemeny L, Abts HF, Ruzicka T. Demonstration and functional analysis of IL-10 receptors in human epidermal cells: decreased expression in psoriatic skin, down-modulation by IL-8, and up-regulation by an antipsoriatic glucocorticosteroid in normal cultured keratinocytes. J Immunol 1997; 159: 6291 - 6297

${ }^{46}$ Asadullah K, Sterry W, Stephanek K. IL-10 is a key cytokine in psoriasis. Proof of principle by IL-10 therapy: a new therapeutic approach. J Clin Invest 1998; 101: 783-794

${ }^{47}$ Ghoreschi K, Thomas P, Dugas M, Mailhammer R, van Eden W, van der Zee R, Biedermann T, Prinz J, Mack M, Mrowietz U, Christophers E, Schlöndorff D, Plewig G, Sander CA, Röcken M. Interleukin-4 therapy of psoriasis induces $\mathrm{Th} 2$ responses and improves human autoimmune disease. Nature Med 2003; 9: 40-46

${ }^{48}$ Ghoreschi K, Röcken M. Immune deviation strategies in the therapy of psoriasis. Curr Drug Targets Inflamm Allergy 2004; 3: 193 - 198

${ }^{49}$ Asadullah K, Volk HD, Sterry W. Novel immunotherapies for psoriasis. Trends Immunol 2002; 23: 47-53

${ }^{50}$ Homey B. Chemokines and chemokine receptors as targets in the therapy of psoriasis. Curr Drug Targets Inflamm Allerg 2004; 3: 169-174

${ }^{51}$ Campbell JJ, Haraldsen G, Pan J, Rottman J, Qin S, Ponath P, Andrew DP, Warnke R, Ruffing N, Kassam N, Wu L, Butcher EC. The chemokine receptor CCR4 in vascular recognition by cutaneous but not intestinal memory T cells. Nature 1999; 400: 776-780

${ }^{52}$ Homey B, Alenius H, Müller A, Soto H, Bowman EP, Yuan W, McEvoy L, Lauerma AI, Assmann T, Bünemann E, Lehto M, Wolff H, Yen D, Marxhausen H, To W, Sedgwick J, Ruzicka T, Lehmann P, Zlotnik A. CCL27-CCR10 interactions regulate T cell-mediated skin inflammation. Nature Med 2002; 8: 157-165

${ }^{53}$ Goebeler M, Toksoy A, Spandau U, Engelhardt E, Bröcker E-B, Gillitzer $\mathrm{R}$. The C-X-C chemokine Mig is highly expressed in the papillae of psoriatic lesions. J Pathol 1998; 184: 89-95

${ }^{54}$ Liao F, Rabin RL, Yannelli JR, Koniaris LG, Vanguri P, Farber JM. Human Mig chemokine: biochemical and functional characterization. J Exp Med 1995; 182: $1301-1314$

${ }^{55}$ Raychaudhuri SP, Jiang WY, Farber EM, Schall TJ, Ruff MR, Pert CB. Upregulation of RANTES in psoriatic keratinocytes: a possible pathogenic mechanism for psoriasis. Acta Derm Venereol 1999; 79: 9-11
${ }^{56}$ Vestergaard C, Gesser B, Lohse N, Jensen SL, Sindet-Pedersen S, Thestrup-Pedersen K, Matsushima K, Larsen CG. Monocyte chemotactic and activating factor (MCAF/MCP-1) has an autoinductive effect in monocytes, a process regulated by IL-10. J Dermatol Sci 1997; 15: $14-22$

${ }^{57}$ Schröder JM, Gregory H, Young J, Christophers E. Neutrophil-activating proteins in psoriasis. J Invest Dermatol 1992; 98: 241 - 247

${ }^{58}$ Schön MP, Zollner TM, Boehncke WH. The molecular basis of lymphocyte recruitment to the skin: Clues for pathogenesis and selective therapies of inflammatory disorders. J Invest Dermatol 2003; 121: 951 962

${ }^{59}$ Dewing SB. Remission of psoriasis associated with cutaneous nerve section. Arch Dermatol 1971; 104: 220-221

${ }^{60}$ Perlman HH. Remission of psoriasis vulgaris from the use of nerveblocking agents. Arch Dermatol 1972; 105: 128-129

${ }^{61}$ Scholzen TE, Luger TA. Neutral endopeptidase and angiotensin-converting enzyme - key enzymes terminating the action of neuroendocrine mediators. Exp Dermatol 2004; 13s : 22s - 26s

62 Raychaudhuri SP, Raychaudhuri SK. Role of NGF and neurogenic inflammation in the pathogenesis of psoriasis. Prog Brain Res 2004; 146: $433-437$

${ }^{63}$ Steinhoff M, Ständer S, Seeliger S, Ansel JC, Schmelz M, Luger TA. Modern aspects of cutaneous neurogenic inflammation. Arch Dermatol 2003; 139: $1479-1488$

${ }^{64}$ Raychaudhuri SP, Sanyal M, Weltman H, Kundu-Raychaudhuri S. K252a, a high-affinity nerve growth factor receptor blocker, improves psoriasis: an in vivo study using the severe combined immunodeficient mouse-human skin model. J Invest Dermatol 2004; 122: 812 819

${ }^{65}$ Robert C, Kupper TS. Inflammatory skin diseases, T cells, and immune surveillance. N Engl J Med 1999; 341: 1817 - 1828

${ }^{66}$ Lebwohl M. Psoriasis. Lancet 2003; 361: $1197-1204$

${ }^{67}$ Papp K, Bissonnette R, Krueger JG, Carey W, Gratton D, Gulliver WP, Lui H, Lynde CW, Magee A, Minier D, Ouellet JP, Patel P, Shapiro J, Shear NH, Kramer S, Walicke P, Bauer R, Dedrick RL, Kim SS, White M, Garovoy MR. The treatment of moderate to severe psoriasis with a new anti-CD11a monoclonal antibody. J Am Acad Dermatol 2001; 45: $665-674$

${ }^{68}$ Butcher EC, Picker LJ. Lymphocyte homing and homeostasis. Science 1996; 272: 60-66

${ }^{69}$ Andrian UH von, Mackay CR. T-cell function and migration. Two sides of the same coin. N Engl J Med 2000; 343: 1020-1034

${ }^{70}$ Ley K. Functions of selectins. Results Probl Cell Differ 2001; 33: 177 200

${ }^{71}$ Fuhlbrigge RC, Kieffer JD, Armerding D, Kupper TS. Cutaneous lymphocyte antigen is a specialized form of PSGL-1 expressed on skin-homing T cells. Nature 1997; 389: $978-981$

72 Bhushan M, Bleiker TO, Ballsdon AE, Allen MH, Sopwith M, Robinson MK, Clarke C, Weller RP, Graham-Brown RA, Keefe M, Barker JN, Griffiths CE. Anti-E-selectin is ineffective in the treatment of psoriasis: a randomized trial. Br J Dermatol 2002; 146: $824-831$

${ }^{73}$ Hardtke M, Friedrich M, Philipp S, Holzmann R, Mueller C, Soos N, Höflich C, Sabat R, Merk H, Sterry W, Mrowietz U. Anti-L-selectin therapy is not effective in psoriasis: a randomized trial (abstr.). Arch Dermatol Res 2005; 296: 427

${ }^{74}$ Boehncke W-H, Schön MP. Interfering with leukocyte rolling - a promising therapeutic approach in inflammatory skin disorders? Trends Pharmacol Sci 2003; 24: 49-52

${ }^{75}$ Friedrich M, Bock D, Philipp S, Ludwig N, Sabat R, Wolk K, SchroeterMaas S, Aydt E, Kang S, Dam TN, Zahlten R, Sterry W, Wolff G. Pan-selectin antagonism improves psoriasis manifestation in mice and man. Arch Dermatol Res 2006; 297: 345 - 351

${ }^{76}$ Schön MP, Drewniok C, Boehncke WH. Targeting selectin functions in the therapy of psoriasis. Curr Drug Targets Inflamm Allerg 2004; 3 : $163-168$

77 Schön MP, Krahn T, Schön M, Rodriguez M-L, Antonicek H, Schultz JE, Ludwig RJ, Zollner TM, Bischoff E, Bremm K-D, Schramm M, Henninger K, Kaufmann R, Gollnick HPM, Parker CM, Boehncke W-H. Efomycine $\mathrm{M}$, a new specific inhibitor of selectin, impairs leukocyte adhesion and alleviates cutaneous inflammation. Nature Med 2002; 8: 366-372

78 Ulbrich H, Eriksson EE, Lindbom L. Leukocyte and endothelial cell adhesion molecules as targets for therapeutic interventions in inflammatory disease. Trends Pharmacol Sci 2003; 24: 640-647

${ }^{79}$ Hwang ST. Mechanisms of T cell homing to skin. Adv Dermatol 2001; 17: $211-241$ 
${ }^{80}$ Ludwig RJ, Zollner TM, Santoso S, Hardt K, Gille J, Baatz H, Johann PS, Pfeffer J, Radeke HH, Schön MP, Kaufmann R, Boehncke WH, Podda M. Junctional adhesion molecules (JAM)-B and -C contribute to leukocyte extravasation to the skin and mediate cutaneous inflammation. J Invest Dermatol 2005; 125: 969-976

${ }^{81}$ Nakada MT, Amin K, Christofidou-Solomidou M, O’Brien CD, Sun J, Gurubhagavatula I, Heavner GA, Taylor AH, Paddock C, Sun QH, Zehnder JL, Newman PJ, Albelda SM, DeLisser HM. Antibodies against the first Ig-like domain of human platelet endothelial cell adhesion molecule-1 (PECAM-1) that inhibit PECAM-1-dependent homophilic adhesion block in vivo neutrophil recruitment. J Immunol 2000; 164: 452 462

${ }^{82}$ Dustin ML, Singer KH, Springer T. Adhesion of T lymphoblasts to epidermal keratinocytes is regulated by interferon gamma and is mediated by intercellular adhesion molecule-1. J Exp Med 1988; 167: 1323 1340

${ }^{83}$ Griffiths CEM, Voorhees JJ, Nickoloff BJ. Characterization of intercellular adhesion molecule- 1 and HLA-DR expression in normal and inflamed skin: Modulation by recombinant gamma interferon and tumor necrosis factor. J Am Acad Dermatol 1989; 20: 617-629

${ }^{84}$ Sterry W, Mielke V, Konter U, Kellner I, Boehncke W-H. Role of $\beta 1$ integrins in epidermotropism of malignant T cells. Am J Pathol 1992; 141: $855-860$

${ }^{85}$ Cepek KL, Shaw SK, Parker CM, Russell GJ, Morrow JS, Rimm DL, Brenner MB. Adhesion between epithelial cells and T lymphocytes mediated by E-cadherin and the alphaEbeta7 integrin. Nature 1994; 372: $190-193$
${ }^{86}$ Pauls K, Schön M, Kubitza RC, Homey B, Wiesenborn A, Lehmann P, Ruzicka T, Parker CM, Schön MP. Role of integrin $\alpha E(C D 103) \beta 7$ for tissue-specific epidermal localization of CD8+ T lymphocytes. J Invest Dermatol 2001; 117: 569-575

${ }^{87}$ Rottman JB, Smith TL, Ganley KG, Kikuchi T, Krueger JG. Potential role of the chemokine receptors CXCR3, CCR4, and the integrin aEb7 in the pathogenesis of psoriasis vulgaris. Lab Invest 2001; 81: 335 - 347

88 Zenz R, Eferl R, Kenner L, Florin L, Hummerich L, Mehic D, Scheuch H, Angel P, Tschachler E, and Wagner EF. Psoriasis-like skin disease and arthritis caused by inducible epidermal deletion of Jun proteins. Nature 2005; 437: 369-375

${ }^{89}$ Sano S, Chan KS, Carbajal S, Clifford J, Peavey M, Kiguchi K, Itami S, Nickoloff BJ, DiGiovanni J. Stat3 links activated keratinocytes and immunocytes required for development of psoriasis in a novel transgenic mouse model. Nat Med 2004; 11: $43-49$

${ }^{90}$ Holick MF. Is psoriasis a T cell disease? Commentary 6. Exp Dermatol 2000; 9: $374-375$

${ }^{91}$ Holick MF, Chimeh FN, Ray S. Topical PTH $(1-34)$ is a novel, safe and effective treatment for psoriasis: a randomized self-controlled trial and an open trial. Br J Dermatol 2003; 149: 370-376

${ }^{92}$ Schröder JM. Is psoriasis a T-cell disease? Viewpoint 2. Exp Dermatol 2000; 9: 363-366

${ }^{93}$ Nestle FO, Conrad C, Tun-Kyi A, Homey B, Gombert M, Boyman O, Burg G, Liu YJ, Gilliet M. Plasmacytoid predendritic cells initiate psoriasis through interferon-alpha production. J Exp Med 2005; 202: 135-143

\section{Buchbesprechung}

\section{Praktische Phlebologie. Empfehlungen zur differenzierten Diagnostik und Therapie phlebologischer Krankheitsbilder E. Rabe, H. E. Gerlach (Hrsg.)}

Stuttgart: Thieme, 2006. 2. Auflage, 156 S., 174 Abb. Geb. 79,95€, ISBN 3-13-119232-1

Die Entwicklungen der letzten Jahre im Bereich der Phlebologie haben nach nur fünf Jahren eine Neuauflage dieses Buches nötig gemacht. Unverändert geblieben ist das Herausgeber-Team: Prof. Rabe aus Bonn und Dr. Gerlach aus Mannheim, beide Vorstandsmitglieder der deutschen Gesellschaft für Phlebologie.

Epidemiologie, Anatomie, Physiologie, Diagnostik, Therapie, eine Übersicht über die gängigen phlebologischen Krankheitsbilder und Begutachtungsfragen sind die im Buch behandelten Themen, eine komplette Übersicht über die Phlebologie.

Das Buch ist gut verständlich und klar geschrieben, die Reihenfolge der Kapitel logisch aufbauend. Die Gliederung ist übersichtlich mit blauen Abhebungen, Wesentliches ist in grau hinterlegten Kästen hervorgehoben. In den Text integriert finden sich fast siebzig gut verständliche Tabellen. Über 170 Bilder und Abbildungen in schwarz/weiß und Farbe verdeutlichen einprägsam Untersuchungstechniken oder Befunde. Eine große Anzahl Phlebographien, Varikographien und Angiographien illustriert diese heutzutage seltener werdende Untersuchungstechnik, mit deren Interpretation häufig Schwierigkeiten auftreten. Die dar- gestellten Hautbefunde sind prägnant und ermöglichen eine klare Vorstellung der im Text beschriebenen Krankheitsbilder. Die Herangehensweise sowohl an die theoretischen als auch die praktischen Lerninhalte ist sehr praxisnah und auf klinische Umsetzung angelegt. Zur Vertiefung finden sich am Ende der Kapitel weiterführende Literaturangaben.

Eingang in das Buch haben auch neue Techniken wie endovaskuläre Laser- oder Radiowellentherapie sowie die Schaumsklerosierung größerer Varizen gefunden. Die Darstellung der Slerosierungstherapie erfolgt im Rahmen der aktuellen Leitlinie der Deutschen Gesellschaft für Phlebologie. Das gesamte Buch ist im Vergleich zur Vorauflage überarbeitet und aktualisiert.

Das Buch bietet einen kompakten Einstieg in die Phlebologie und vermittelt für Einsteiger alle notwendigen Grundlagen sowie das klinische Basiswissen in gut verständlicher und klarer Form. Das Buch ist aufgrund seiner Gliederung, seines Aufbaus und seines Stils gut zu lesen, die Vermittlung des Wissens gelingt nebenbei. Wer tiefer in die Materie eintauchen will, benötigt gezielt weiterführende Literatur, z. B. in Form von speziellen Operationslehren. Das Buch wird seinem Namen „Praktische Phlebologie“ voll und ganz gerecht. Für Neueinsteiger bietet es einen guten Überblick, für andere ist es eine knappe Auffrischung des Basiswissens. Knapp, verständlich, einfach praktisch.

W. Koenen, Mannheim 